\title{
ACCRETION OF MATTER ONTO BLACK HOLES
}

\author{
R. A. SUNYAEV \\ Institute of Applied Mathematics, Moscow, U.S.S.R.
}

(Presented by M. M. Basko)

The theory of disk accretion is presented in the paper by Shakura and Sunyaev (1973). The structure of the disk, its luminosity and the spectrum produced are discussed. The reported quasiperiodic variability in the radiation from black holes is analysed in the paper by Sunyaev (1972). Reflection of X-rays and the possibility of observing $\mathrm{X}$-ray pulsars which do not have a beam impinging on the Earth was considered by Basko et al. (in press).

The beaming of radiation by the accretion onto magnetic neutron stars is reported by Gnedin and Sunyaev (1973) and Basko and Sunyaev (in press). The beamed optical radiation from neutron stars due to the gyroemission of heavy ions and its connection with the observed optical pulsations of $\mathrm{HZ}$ Her are discussed by Gnedin and Sunyaev (in press). They also propose to search for the X-ray line in the spectrum of X-ray pulsars which corresponds to the electron gyrofrequency. In the paper by Cherepashchuk and Sunyaev (in press), new arguments in favor of the mass Cyg X-1 exceeding $3 M_{\odot}$ are presented. The properties of other X-ray binary systems are discussed.

\section{References}

Basko, M. M. and Sunyaev, R. A.: Astron. Astrophys., in press.

Basko, M. M., Sunyaev, R. A., and Titarchuk, L. G.: Astron. Astrophys., in press.

Cherepashchuk, A. M. and Sunyaev, R. A.: Monthly Notices Roy. Astron. Soc., in press.

Gnedin, Yu. N. and Sunyaev, R. A.: 1973, Astron. Astrophys 25, 233.

Gnedin, Yu. N. and Sunyaev, R. A.: Astrophys. Space Sci., in press.

Shakura, A. I. and Sunyaev, R. A.: 1973, Astron. Astrophys. 24, 337.

Sunyaev, R. A.: 1972, Astron. Zh. 49, 1153. 